\title{
Tomography as a diagnostic tool for phase space mapping of intense particle beams
}

\author{
D. Stratakis, R. A. Kishek, H. Li, ${ }^{*}$ S. Bernal, M. Walter, B. Quinn, M. Reiser, and P. G. O'Shea \\ Institute for Research in Electronics and Applied Physics, University of Maryland, College Park, Maryland 20742, USA
}

(Received 3 April 2006; published 7 November 2006)

\begin{abstract}
A technique is described for the tomographic mapping of transverse phase space in beams with space charge. Most prior studies where performed at high energy where space charge was negligible and therefore not considered in the analysis. The tomographic reconstruction process is compared with results of simulations using the particle-in-cell code WARP. The new tomographic technique is tested for beams with different intensities (both emittance and space-charge dominated), and with different initial distributions. Effects of various errors in the data collection process on the reconstructed phase space are discussed. It is shown that the crucial factor is not necessarily the number of projections but the range of angles over which the projections are taken. This study also includes a number of experimental results on tomographic phase space mapping performed on the University of Maryland Electron Ring.
\end{abstract}

DOI: 10.1103/PhysRevSTAB.9.112801

PACS numbers: 41.85.- p, 41.75.Fr, 52.59.Sa

\section{INTRODUCTION}

Intense particle beams have applications in many different research areas which impact our lives. Such applications of intense beams include accelerator-driven neutron sources [1], higher-luminosity high-energy colliders, free electron lasers (FEL) [2], and heavy-ion inertial fusion drivers (HIF) [3]. Neutron sources and FELs have important applications in material research, helping us better understand the structure of matter and improving the quality of materials. Free electron lasers contribute to the processing of components for electronics, microtechnology, and nanotechnology. Finally, heavy-ion inertial fusion drivers promise the production of large and unlimited amounts of energy which can be harnessed to provide an affordable and environmentally attractive source of electrical power. All these applications are premised on the considerable challenge of generating, transporting, accelerating, and focusing large amounts of particles confined in a narrow region of phase space, without significant particle losses or deterioration of beam quality. One nanocoulomb of charge confined into a $300 \mathrm{fs}$ bunch (line charge density equal to $11 \mu \mathrm{C} / \mathrm{m}$ ) with $1 \mu \mathrm{m}$ transverse normalized emittance are typical parameters for such beams, with HIF drivers demanding much more charge per bunch.

In order to study the dynamics of such intense beams in a cost efficient way, we have designed and recently commissioned the University of Maryland Electron Ring (UMER) [4]. Using low energy ( $<10 \mathrm{keV}$ ) and high current (up to $100 \mathrm{~mA}$ ), electron beams with extreme intensities can be produced [5]. Some challenging scientific issues arise from the space charge and collective behavior effects in such high current beams [6]. These include emittance growth, halo formation, collective instabilities, and $x-y$ energy transfer and coupling. To investigate these effects, knowl-

*Present address: Microsoft Corp., Redmond, WA 98052. edge of the actual phase space distributions and emittances of the beam in certain locations is required.

Standard methods [7] that are commonly used in the accelerator community to map the beam phase space are the quadrupole scan, pepper pot, and slit wire scan. In the quadrupole scan technique, the Courant-Snyder parameters can be obtained by measuring the beam size at a given point some distance from a quadrupole magnet, as a function of focusing strengths. The technique has two important limitations: First, in the analysis we make the usual assumption that the transverse phase space distribution fills an ellipse; and second, the method becomes questionable when space charge cannot be neglected [8]. Pepper pots measure the phase space distribution by using apertures to localize specific regions in transverse space. The beam is intercepted by a screen, or pepper-pot plate, which is normal to the beam and contains a regular array of identical holes over its entire surface. In order to get a good resolution of the reconstructed phase, a sufficient number of beamlets should be produced. However, when the beam size is small, as (for instance) in the UMER $0.6 \mathrm{~mA}$ beam which is of the order of mm, very small and closely spaced holes are needed. In this regime, construction of such a mask would pose serious practical difficulties. Finally, in slit-based emittance measurements, the beam is collimated by a set of slits scanned across the beam, and then the collimated beamlets drift a given length and finally are analyzed by a collector upstream. The advantage of the technique is that it allows time resolved measurements; however, a serious problem is the limited signal to noise ratio of the beam signal after passing the slit scanners.

Computerized tomography [9] is well known in the medical community and was originally developed to process $\mathrm{x}$-ray images. Tomography is related to a theorem by Radon [10], who stated that an object in $n$-dimensional space can be recovered from a sufficient number of projections onto $(n-1)$-dimensional space. In the physics of beams it is possible to use tomographic image reconstruc- 
tions to map the phase space by measuring spatial projections of the electron beam. Over the past decade, phase space tomography has been used successfully in relativistic electron beams without space charge [11-13] and recently applied to beams with space charge [14], by assuming linear forces. Additionally, the idea of reconstructing an image from projections was further extended to map the 4D distribution $f\left(x, y, x^{\prime}, y^{\prime}\right)$, for beams with space charge [15].

Even though tomography has been used over the past years as a diagnostic to map the beam phase space, some questions remain about the limitations of the method. Can the technique be used for beams with space charge? Could a simple linear space charge approximation accurately reproduce the beam phase space? In addition, will tomography map the phase space for beams with different and more complex profiles? For instance, what would happen if the beam starts with a hollow velocity distribution (such as is repeatedly observed on UMER)? In order to answer such questions, we simulate a UMER beam experiment using the particle-in-cell code WARP [16], and apply tomography to reconstruct the phase space. In this way both space charge and input beam distributions can be controlled by the code, and the accuracy of tomography will be examined. The outline of our work is as follows: In Sec. II a short general description of the tomography algorithm will be given. In Sec. III, we will describe our approach for applying tomography to beams. In Sec. IV, we will examine the effect of various errors in the data collection process on the reconstructed phase space (number of projections, rotation angle, and uncertainties in measurements). In Sec. V, the application of the tomographic technique to beams with space charge will be discussed. Finally, in Sec. VI we present experimental results on tomographic phase space mapping on UMER.

\section{TOMOGRAPHY ALGORITHM}

Several algorithms [17] are available to compute high quality reconstructions from projection data, e.g., algebraic reconstruction technique, maximum entropy tomography, filtered-backprojection algorithm (FBA), etc. The FBA algorithm is the most common method to reconstruct a two-dimensional image and it is generally believed that it provides a reconstructed image of high quality with normally available computer capacity and computational times [17].

Suppose that $f(x, y)$ corresponds to a two-dimensional distribution. Then the integral

$$
\hat{f}(\rho, \theta)=\int_{-\infty}^{\infty} \int_{-\infty}^{\infty} d x d y f(x, y) \delta(\rho-x \cos \theta-y \sin \theta)
$$

defines the transverse projection of the distribution $f(x, y)$ along the axis $\rho=x \cos \theta+y \sin \theta$, placed at an angle $\theta$ relative to the $x$-axis. Such a projection is known as the
Radon transform of the function $f(x, y)$. If $F(u, v)$ is the two-dimensional Fourier transform of the function $f(x, y)$, then its inverse Fourier transform is given by

$$
f(x, y)=\int_{-\infty}^{\infty} \int_{-\infty}^{\infty} F(u, v) e^{j 2 \pi(u x+v y)} d u d v .
$$

By exchanging the rectangular coordinate system in the frequency domain $(u, v)$ for a polar coordinate system $(w, \theta)$, Eq. (2) becomes

$$
f(x, y)=\int_{0}^{\pi} \int_{-\infty}^{\infty} F(w, \theta)|w| e^{j 2 \pi w \rho} d w d \theta
$$

Likewise, the Fourier transform of the Radon transform is

$$
S(w, \theta)=\int_{-\infty}^{\infty} \hat{f}(\rho, \theta) e^{-j 2 \pi w p} d \rho .
$$

Using the Fourier slice theorem [17], we can write $F(w, \theta)=S(w, \theta)$ and therefore

$$
f(x, y)=\int_{0}^{\pi} \int_{-\infty}^{\infty} S(w, \theta)|w| e^{j 2 \pi w \rho} d w d \theta,
$$

or simpler

$$
f(x, y)=\int_{0}^{\pi} Q(\rho, \theta) d \theta
$$

where $Q$ often is called “filtered projection" [17]. Therefore, if a number of projections between 0 and $\pi$ are known, the distribution can be reconstructed by backprojecting the filtered version of the projections according to Eq. (6).

In beam physics, we usually measure projections of the 4D phase space onto a 2D configuration-space imager. Our goal is to obtain information on the velocity space distribution by combining the ideas of tomography with the systematic scanning of quadrupole magnets, which induce rotations of the beam in phase space [11]. The idea is the following: Suppose that $\mu\left(x_{0}, x_{0}^{\prime}\right)_{z_{0}}$ is the phase space distribution at a certain location $z_{0}$ in the beam line. If $\mu\left(x_{1}, x_{1}^{\prime}\right)_{z_{1}}$ is the phase distribution at another location $z_{1}$, then by assuming that we have a linear system, the particle motion at the two positions obeys

$$
\left(\begin{array}{c}
x_{1} \\
x_{1}^{\prime}
\end{array}\right)=M_{1}\left(\begin{array}{c}
x_{0} \\
x_{0}^{\prime}
\end{array}\right)
$$

where $M_{1}=\left(\begin{array}{ll}M_{11} & M_{12} \\ M_{21} & M_{22}\end{array}\right)$ is the transport matrix between $z_{0}$ and $z_{1}$. The transport line consists of drift sections and thick quadrupole(s). Thus, $M_{1}$ is a function of the quadrupole current(s).

Integration of the phase space distribution $\mu\left(x_{1}, x_{1}^{\prime}\right)_{z_{1}}$ along $x_{1}^{\prime}$ is equivalent to the beam profile along $x_{1}$ and is given by

$$
C\left(x_{1}\right)=\int \mu\left(x_{1}, x_{1}^{\prime}\right)_{z_{1}} d x_{1}^{\prime} .
$$

Using the Dirac delta function, it can be written in the 
equivalent form

$$
C(x)=\iint \mu\left(x_{1}, x_{1}^{\prime}\right)_{z_{1}} \delta\left(x_{1}-x\right) d x_{1} d x_{1}^{\prime} .
$$

According to Liouville's theorem, the particle density remains constant, therefore

$$
\mu\left(x_{0}, x_{0}^{\prime}\right)_{z_{0}}=\mu\left(x_{1}, x_{1}^{\prime}\right)_{z_{1}} .
$$

Hence, by combining Eq. (9) with Eq. (10), we can write

$$
C(x)=\iint \mu\left(x_{0}, x_{0}^{\prime}\right)_{z_{0}} \delta\left(M_{11} x_{0}+M_{12} x_{0}^{\prime}-x\right) d x_{0} d x_{0}^{\prime} .
$$

In order to relate Eq. (11) to the Radon transform, we define the scaling factor $s$ [11] by

$$
s=\sqrt{M_{11}^{2}+M_{12}^{2}}
$$

and the phase space rotation angle $\theta$ [11] by

$$
\tan \theta=\frac{M_{12}}{M_{11}}
$$

Now, using Eqs. (12) and (13), Eq. (11) becomes

$C(x, \theta)=\frac{1}{s} \iint \mu\left(x_{0}, x_{0}^{\prime}\right)_{z_{0}} \delta\left(x_{0} \cos \theta+x_{0}^{\prime} \sin \theta-\rho\right) d x_{0} d x_{0}^{\prime}$,

where $\rho=x / s$.

Comparing Eq. (1) with Eq. (14) we can write

$$
\hat{f}(\rho, \theta)=s C(x, \theta) \text {. }
$$

From Eq. (15) we can deduce that a simple scaling equation relates the spatial beam projections to the Radon transform, $\hat{f}(\rho, \theta)$, of the transverse phase space. This is a very useful result since the beam spatial distribution can be easily obtained in experiments, e.g., using a phosphor screen. Both scaling factor and angle of the projection can be easily calculated from the beam line overall transport matrix and are functions of the quadrupole focusing. Therefore, any variation of the quadrupole strength can result in a number of phase space projections at various angles that can be used according to Eq. (6) to recover the actual phase space.

\section{EXPERIMENTAL CONFIGURATION AND COMPUTER MODEL}

In our transport line we employ three quadrupoles, $Q 1$, $Q 2$, and $Q 3$ to achieve a full $180^{\circ}$ phase space rotation. Figure 1(a) presents a simplified diagram showing the orientation of these quadrupoles, and Fig. 1(b) shows the actual photo. DP1 and DP2 are bending dipoles which are ignored in the analysis. The beam profiles of a $10 \mathrm{keV}$ beam are obtained by means of a phosphor screen placed at point $z_{1}$ downstream of $Q 3$. We also choose the same position for the phase space to be reconstructed, which implies $z_{0}=z_{1}$. In that way we can directly compare the recovered phase space with the actual spatial distribution. Along with the experimental configuration, the relevant matrix representations are also illustrated in Fig. 1. In our case, the phase space $\mu\left(x_{0}, x_{0}^{\prime}\right)_{z_{0}}$ to be reconstructed is the one where $Q 1$ is operated at $I_{1}=1.884 \mathrm{~A}$ and $Q 2$ and $Q 3$ are turned off. Therefore, the inverse of the matrices $D_{0}, M_{Q 1,0}$ needs to be included in the transfer matrix analysis where

$$
D_{0}=\left(\begin{array}{cc}
1 & L \\
0 & 1
\end{array}\right)
$$

is the drift matrix and

$$
M_{Q 1,0}=\left(\begin{array}{cc}
\cos \sqrt{\kappa} z & \frac{1}{\sqrt{\kappa}} \sin \sqrt{\kappa} z \\
-\sqrt{\kappa} \sin \sqrt{\kappa} z & \cos \sqrt{\kappa} z
\end{array}\right)
$$

is the quadrupole matrix; $\kappa$ is the focusing strength in $m^{-2}$ of the quadrupole corresponding to the current $I_{1}$.

If $M_{Q 1}, M_{Q 2}, M_{Q 3}$ are the matrices of $Q 1, Q 2$, and $Q 3$, then the overall transport matrix, $M_{1}$, from $z_{0}$ to $z_{1}$ will be

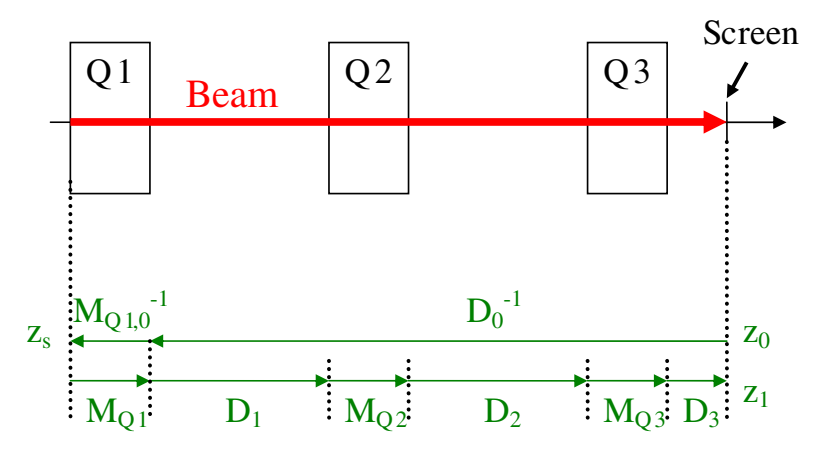

(a)

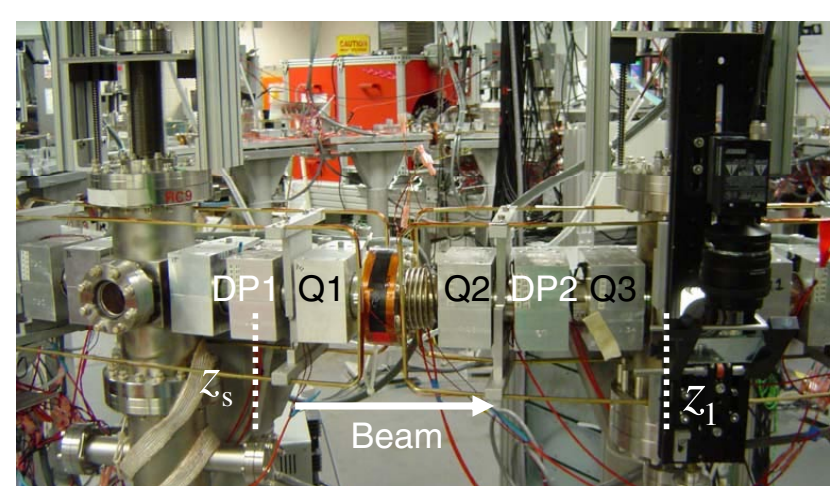

(b)

FIG. 1. (Color) Tomography experimental configuration: (a) simplified diagram; (b) actual experimental setup. 


$$
M_{1}=D_{3} M_{Q 3} D_{2} M_{Q 2} D_{1} M_{Q 1}\left(D_{0} M_{Q 1,0}\right)^{-1} .
$$

In the presence of space charge, calculation of transport matrix elements becomes more complicated. In order to simplify the analysis, some assumptions need to be made about the density distribution and the resulting space charge forces. One method that has been proposed [14] is to use linear space charge forces, which are estimated from the beam envelopes, to correct the transfer matrices between different locations. Its accuracy will be analyzed in the computer simulation, as shown later in this paper. The detailed process is as follows: Assuming linear space charge forces, the net focusing strengths become [6]

$$
\begin{gathered}
\kappa_{x}=\kappa_{x 0}-\frac{2 K}{X(X+Y)} \\
\kappa_{y}=\kappa_{y 0}-\frac{2 K}{Y(X+Y)},
\end{gathered}
$$

where $\kappa_{x 0}, \kappa_{y 0}$ are quadrupole focusing strengths and $X, Y$ are the $2 x_{\text {rms }}$ beam envelope sizes for $x$ and $y$ directions, respectively. In our analysis, for the low intensity beam $(0.6 \mathrm{~mA})$, only the terms $\kappa_{x 0}, \kappa_{y 0}$ were used in generating the transfer matrices; however, for a more intense beam the defocusing space charge terms $-\frac{2 K}{X(X+Y)}$ and $-\frac{2 K}{Y(X+Y)}$ must be included in the matrix analysis.

In order to obtain the net focusing strength, knowledge of the beam sizes $X$ and $Y$ is needed. Calculation of the beam size is not easy since it is a function of $z$ and the quadrupole focusing function also. Looking over the experimental setup in Fig. 1, we do not have any diagnostic over the distance crossing the three quadrupoles to get any information of the evolution of $X$ and $Y$. However, assuming no emittance growth we can calculate the beam envelopes using the Kapchinskij-Vladimirskij envelope equations [6] given by

$$
\begin{aligned}
& X^{\prime \prime}+\kappa_{x} X-\frac{2 K}{X+Y}-\frac{\varepsilon_{x}^{2}}{X^{3}}=0 \\
& Y^{\prime \prime}+\kappa_{y} Y-\frac{2 K}{X+Y}-\frac{\varepsilon_{y}^{2}}{Y^{3}}=0
\end{aligned}
$$

starting with estimated initial beam sizes and slopes before the first quadrupole. We can check our assumptions by comparing the calculated and measured beam sizes at the phosphor screen location. In case they do not agree well, we adjust the initial conditions and repeat our envelope calculations until we get agreement.

Once the evolution of $X, Y$ with respect to $z$ is known, we can calculate the net focusing functions given by Eqs. (19) and (20). The new transfer matrix can be modeled by the superposition of many hard edge subelements. Finally, from Eqs. (12) and (13) we can calculate the rotation angle and scaling factor.
We simulated the above experimental setup with the particle-in-cell code WARP [16], developed at the Lawrence Livermore National Laboratory. The advantage of WARP is that it self-consistently includes space charge effects and has been successfully benchmarked against UMER experimental data [18]. For each quadrupole current setting, we run a WARP simulation, collect a snapshot of the beam density in configuration space at the phosphor screen location, and then process it to look like a phosphor screen image. The quadrupole scan results in a large number of photos, each associated with a different projection, and hence a different phase space rotation angle and scaling factor, as defined from the transfer matrix by using Eqs. (12) and (13). For image processing we developed a code based on PYTHON. The PYTHON code processes each photo to obtain profiles along $x$ and $y$, and then scales them to obtain the Radon transform of the phase space distribution. Operating on a number of projections, the code uses the algorithm described in Sec. II to recover the original phase space distribution.

While scanning the quadrupoles there are some constraints that we need to keep in mind, especially when doing experiments. First, the beam at the measurement point must remain within the phosphor screen. Second, the beam must be well aligned throughout the transport section to eliminate image-charge effects. Third, the quadrupole current cannot exceed \pm 3.5 A due to the possible overheating and power supply limitations.

In summary, in order to reconstruct phase space we use the following process: (1) we generate a table containing the desired quadrupole current settings and the corresponding rotation angles and scaling factors; (2) repeatedly run the simulation code and obtain a beam photo at the phosphor screen location for each setting; (3) process the images using the PYTHON code to calculate the beam profile by integration for each photo; (4) calculate the modified projection $Q(\rho, \theta) ;(5)$ integrate the filtered projection over all rotation angles using Eq. (6).

\section{ERROR ANALYSIS}

When using tomography to reconstruct beam phase spaces several questions arise. For instance, how accurate is the FBA algorithm? How many projections are needed? What happens when the phase space rotation is less than $180^{\circ}$ ? How sensitive is the reconstruction to uncertainties in the input parameters? To answer such questions we model a quadrupole scan for a low current electron beam and follow the process described in Sec. III to tomographically reconstruct the phase space. The tomographyreconstructed phase space is compared to the phase space generated directly by WARP. The phase space generated by WARP does not make the assumptions that tomography does (constant emittance, linear forces, no image forces) and therefore can be used as a prototype to establish the quality of our tomography method. For the simulation, we use a 
low-current $(0.6 \mathrm{~mA})$, nonrelativistic $(10 \mathrm{keV})$ electron beam. We use a $512 \times 512$ grid for the Poisson solver, a step size of $2 \mathrm{~mm}$ along $z$, and 64000 particles. Running a large number of test simulations with more particles or higher resolution resulted in no perceptible difference in the final result.

Figure 2 shows reconstructed phase spaces by tomography when a full 180 rotation is achieved but a different number of projections is used. Figure 2(d) depicts recovery for 210 projections. Figure 2(c) shows recovery for 105 projections (every other one) and Fig. 2(b) for 70 (every third one). Finally, for Fig. 2(a) only 30 projections where used (every fifth one). The near absence of streaking artifacts in Fig. 2(d) indicates that 210 projections are enough for complete recovery.

Figure 3 compares the above phase space constructed by tomography [Fig. 3(a)] to that generated directly by WARP [Fig. 3(b)]. From the phase space distribution we are able to calculate the effective emittances by using the following relation:

$$
\varepsilon_{\mathrm{eff}}=4 \varepsilon_{\mathrm{rms}}=4 \sqrt{\left\langle x^{2}\right\rangle\left\langle x^{\prime 2}\right\rangle-\left\langle x x^{\prime}\right\rangle^{2}}
$$

and we get $\varepsilon_{\text {eff }}=5.4 \mu \mathrm{m}$, and $\varepsilon_{\text {eff }}=5.5 \mu \mathrm{m}$ for the direct WARP and tomography processed phase space, respectively. Thus, as far as calculating the emittance of a low-current beam, tomography exhibits excellent agreement, resulting in a prediction within $1.8 \%$ of the actual

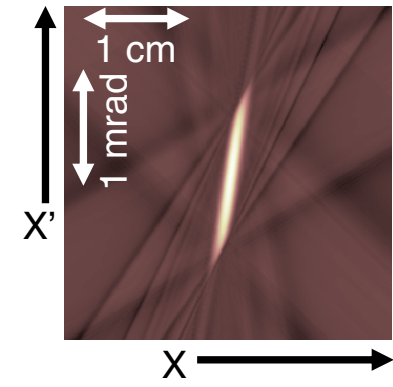

(a)

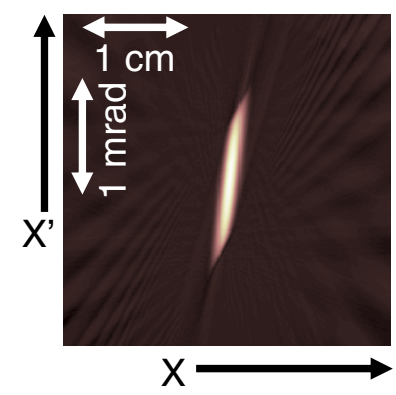

(c)

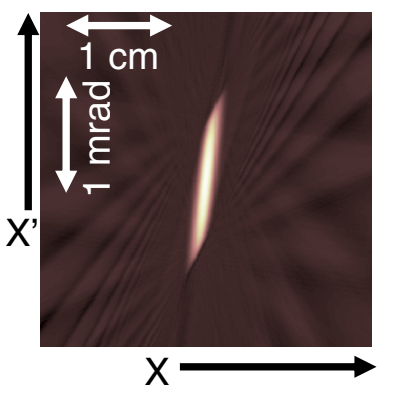

(b)

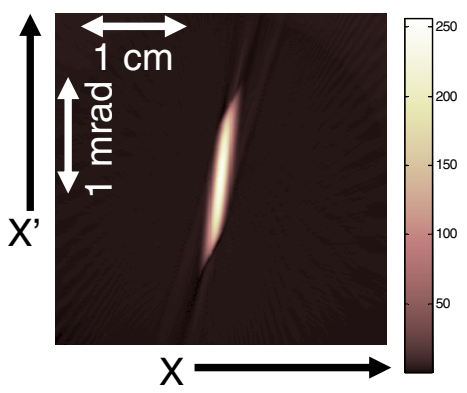

(d)

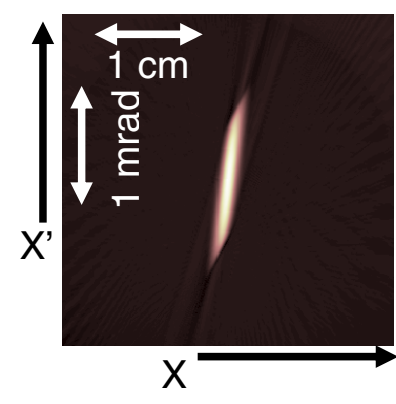

(a)

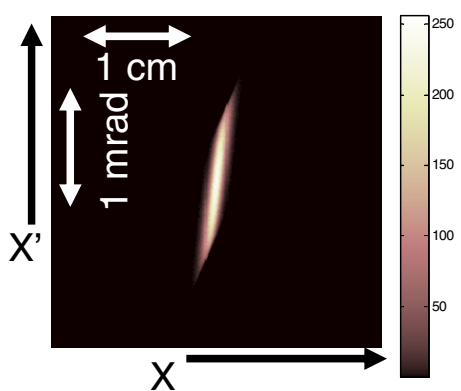

(b)
FIG. 3. (Color) $X^{\prime} X$ phase space for the $0.6 \mathrm{~mA}$ electron beam: (a) using tomography; (b) direct WARP.

value. In order to verify the recovered phase space, we calculate the beam size from the phase space distribution and compare it with the size obtained from the spatial distribution at the same point. The results are $2 x_{\mathrm{rms}}=$ $1.26 \mathrm{~mm}$ from the phase space and $2 x_{\mathrm{rms}}=1.30 \mathrm{~mm}$ from the spatial distribution.

Next, we are interested in performing recovery for the case where the total rotation angle is less than $180^{\circ}$. Care was taken that our number of projections was above 105 in order to avoid aliasing effects from the number of projections. Our results are shown in Fig. 4. In Fig. 4(a) recovery is done when the phase space is rotated a total angle of

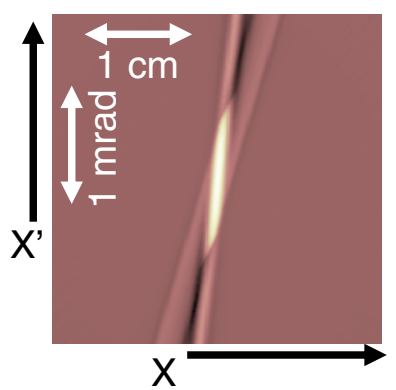

(a)

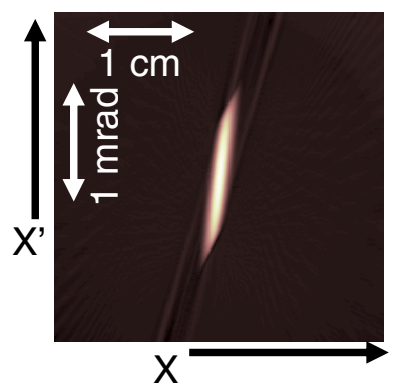

(c)

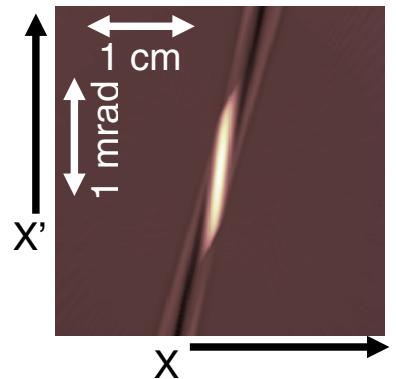

(b)

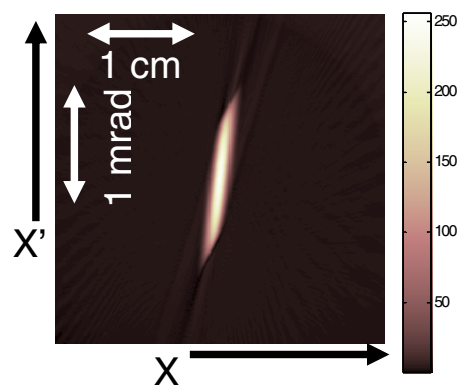

(d)
FIG. 2. (Color) $X^{\prime} X$ tomographic recovery of the $0.6 \mathrm{~mA}$ (pencil beam) using a different number of projections. (a) 30 projections, (b) 70 projections, (c) 105 projections, (d) 210 projections.
FIG. 4. (Color) $X^{\prime} X$ tomographic reconstruction of the pencil beam with different overall angular spans. (a) 170 degrees, (b) 175 degrees, (c) 178 degrees, (d) 180 degrees. 
(a)

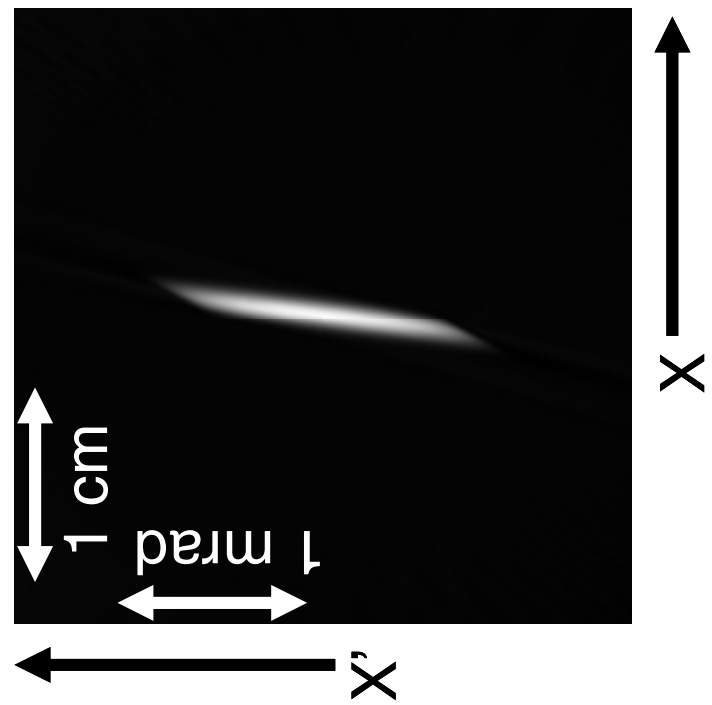

(b)

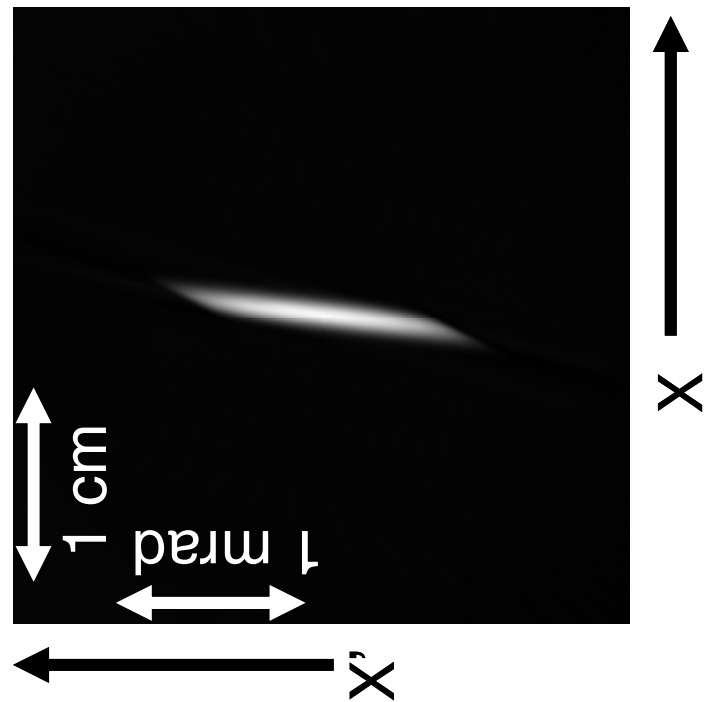

(c)

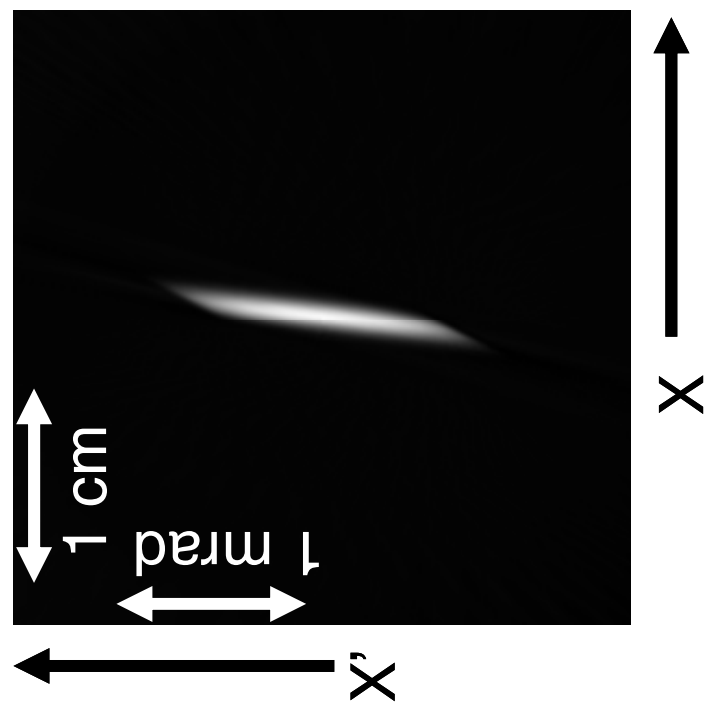

FIG. 5. $X^{\prime} X$ phase space distribution of the pencil beam with different simulated systematic errors. (a) $1 \%$ error, (b) $2 \%$ error, (c) $4 \%$ error. $170^{\circ}$ (127 projections). Figures 4(b) and 4(c) represent recoveries for $175^{\circ}$ (178 projections) and $178^{\circ}$ (192 projections) rotations, respectively. Finally, Fig. 4(d) corresponds to the case where a full $180^{\circ}$ rotation is achieved. We conclude that in order to recover a phase space with minimum background noise we need to collect projections within the full $180^{\circ}$ range.

Finally, since real experiments involve an uncertainty between the actual value and the set value in quadrupole current, we want to address the issue of quadrupole current systematic errors in the phase space recovery process. Figure 5 depicts the recovered electron beam phase space using 210 projections, with (a) $1 \%$ simulated systematic error, (b) $2 \%$ simulated systematic error, and (c) 4\% simulated systematic error. We conclude that the uncertainty in the quadrupole current values will affect the recovered phase space. Quantitatively, if we measure the beam emittance we get 5.6, 5.7, $5.8 \mu \mathrm{m}$ for the cases where we have $2 \%, 3 \%$, and $5 \%$ error, respectively. Comparing these values to the emittance that we found for the case where no uncertainties in currents where present $(5.5 \mu \mathrm{m})$, we conclude that small systematic errors in the quadrupole current have a moderate impact on the resulting emittances.

\section{TOMOGRAPHY FOR SPACE CHARGE DOMINATED BEAMS}

\section{A. Space charge beams and initial distribution}

In UMER, we are able to control the beam current of a $10 \mathrm{keV}$ electron beam by collimation at the gun exit. Beam currents within the $10-100 \mathrm{~mA}$ range can be generated this way. For lower currents, the thermal pressure force is higher than the space charge force and the beams are referred to as emittance dominated. For higher currents, the space charge force exceeds the thermal pressure force and the beams are known as space charge dominated. These two regimes are distinguished by using the intensity parameter $\chi$, which is defined as the ratio of the space charge force relative to the external focusing force [5]. If $0<\chi \leq 0.5$ the beam is emittance dominated; if $0.5<$ $\chi<1$ it is space charge dominated. Having applied tomography successfully for emittance dominated beams in Sec. IV, we then endeavored to extend the technique to beams with space charge.

We start our simulation for a UMER beam with current equal to $I=7 \mathrm{~mA}$ and intensity parameter $\chi=0.72$. Since this is an intense beam, when doing tomography space-charge forces must be taken into consideration; however, as discussed in Sec. III, we will assume that linearity still holds. We begin our simulation with a semi-Gaussian (S-G) initial distribution, which is commonly used for modeling space-charge-dominated beams. In such a distribution the particle density is uniform across the beam, while the velocity profile is Gaussian with uniform temperature. The recovered phase space is dem- 
onstrated in Fig. 6(a). Figure 6(b) represents the phase space generated directly (without tomography) from WARP. Next, we wish to compare emittances calculated from these two phase spaces. From tomography we get $\varepsilon_{\text {eff }}=13.7 \mu \mathrm{m}$ and from WARP simulation $\varepsilon_{\text {eff }}=$ $14.5 \mu \mathrm{m}$. In addition, calculation of the $2 x_{\text {rms }}$ beam sizes from the tomo-recovered phase space and the corresponding spatial distribution (shown on Fig. 7) yields $2 x_{\text {rms }}=$ $1.88 \mathrm{~mm}$ and $2 x_{\mathrm{rms}}=1.80 \mathrm{~mm}$, respectively. For both beam size and emittance values, there is about $5 \%$ difference between the results from tomography and those from WARP.

In beam physics the initial beam density profile is an important factor in determining its evolution. Part of the challenge of any distribution measurement technique is that it should make no a priori assumptions about those initial distributions but instead should be equally accurate for different input profiles. Therefore, instead of using a S$\mathrm{G}$ we model our tomography process for beams starting with a hollow velocity distribution. Such a distribution has

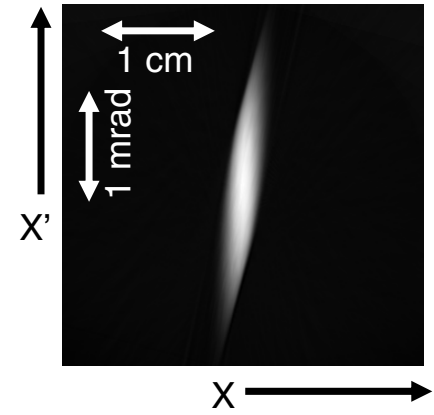

(a)

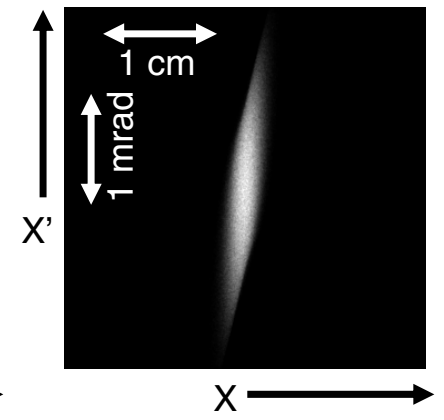

(b)
FIG. 6. $X^{\prime} X$ phase space distribution of the $7 \mathrm{~mA}$ electron beam: (a) by tomography; (b) by direct WARP simulation.

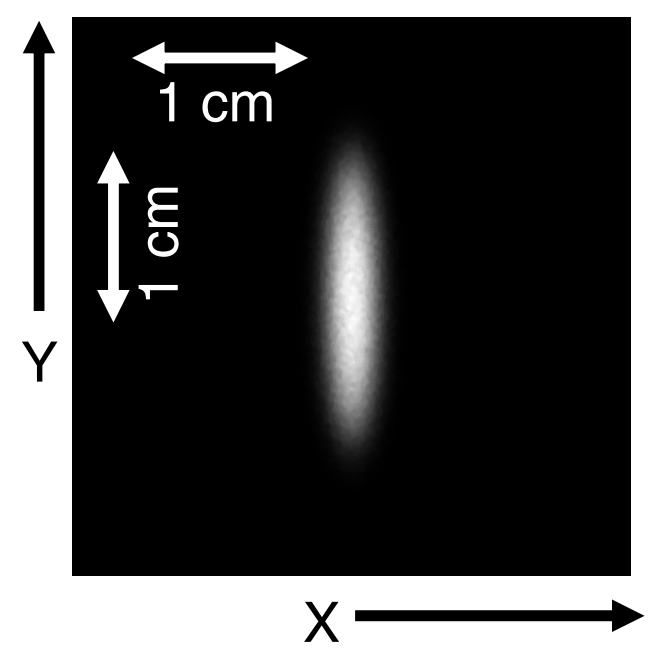

FIG. 7. Spatial distribution of the $7 \mathrm{~mA}$ electron beam starting with a Gaussian velocity distribution. been experimentally observed in UMER [18]. In order to model such a beam profile, we rotate a Gaussian distribution with width $v_{\text {th }}$ and offset $\alpha$ from the origin around a circle of radius $\alpha$ [18]. The parameters $v_{\text {th }}$ and $\alpha$ are obtained empirically such that rms size and emittance of the hollow distribution are the same as the semi-Gaussian distribution in the previous section. In this way both beams are equivalent. It should be noted that the spatial density is still uniform.

Figure 8(a) shows the recovered phase space by tomography and Fig. 8(b) shows the phase space from direct WARP simulation for the $7 \mathrm{~mA}$ beam. Figure 9 illustrates the corresponding spatial distribution, which also displays a hollow structure. From the tomography processed phase space we obtain $\varepsilon_{\text {eff }}=14.1 \mu \mathrm{m}$ and from WARP simulation we get $\varepsilon_{\text {eff }}=14.4 \mu \mathrm{m}$. There is a $2 \%$ difference in the emittance measurements. By comparing the beam sizes from the recovered phase space and the corresponding spatial distribution (shown in Fig. 9), we obtain $2 x_{\text {rms }}=$ $1.85 \mathrm{~mm}$ and $2 x_{\mathrm{rms}}=1.87 \mathrm{~mm}$, respectively. Table I contains a summary of emittance measurements for both pen-
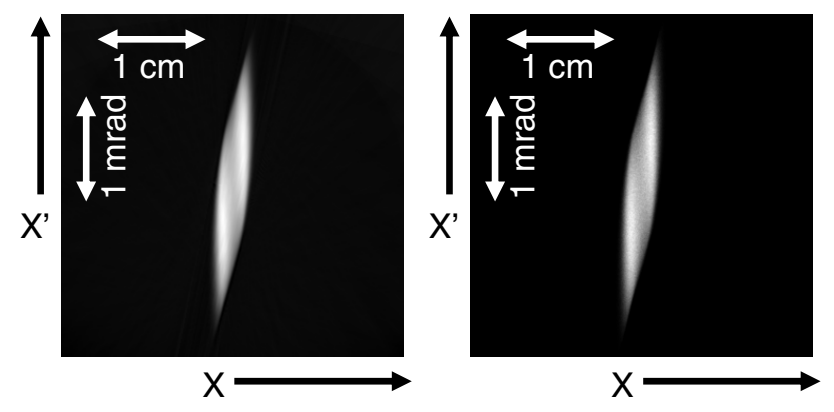

FIG. 8. $X^{\prime} X$ phase space distribution of the $7 \mathrm{~mA}$ electron beam starting with a hollow velocity distribution: (a) by tomography; (b) by direct WARP simulation.

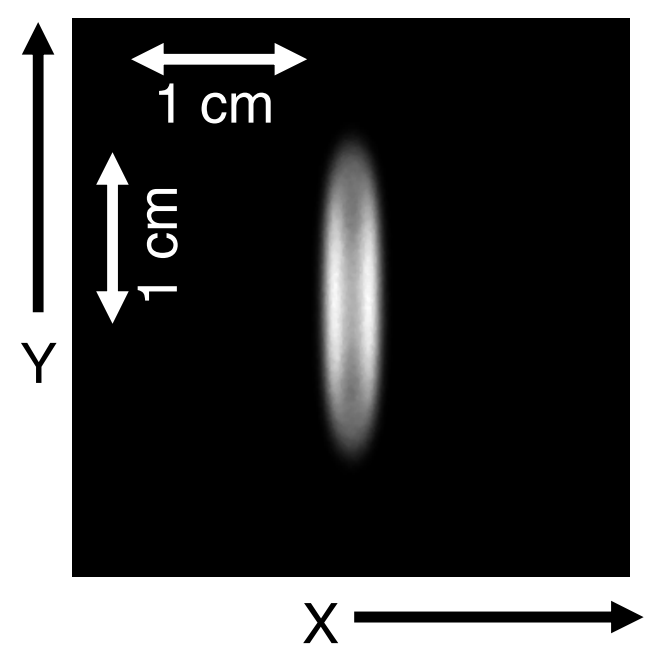

FIG. 9. Spatial distribution of the $7 \mathrm{~mA}$ electron beam starting with a hollow velocity distribution. 
TABLE I. Summary of emittance measurements for different input distributions and beam currents.

\begin{tabular}{lcccc}
\hline \hline \multicolumn{1}{c}{ Distribution } & \multicolumn{2}{c}{ Semi-Gaussian } & \multicolumn{2}{c}{ Hollow velocity } \\
Beam current $(\mathrm{mA})$ & 0.6 & 7 & 0.6 & 7 \\
\hline$\varepsilon_{x}$, WARP $(\mu \mathrm{m})$ & 5.4 & 14.5 & 5.5 & 14.4 \\
$\varepsilon_{x}$, Tomo $(\mu \mathrm{m})$ & 5.5 & 13.7 & 5.8 & 14.1 \\
$\%$ error & 1.8 & 5.5 & 5.5 & 2 \\
\hline \hline
\end{tabular}

cil and $7 \mathrm{~mA}$ beams under different initial distributions obtained by WARP and tomography.

\section{B. Transverse density waves}

Beam particle distributions normally reach an equilibrium state only after traversing a long distance in a linear transport system, or after many turns in a circular machine. Thus, beams are typically far from equilibrium near their source. Many factors contribute to the beam evolution towards equilibrium, causing emittance growth in the process: envelope mismatch, nonlinearities and anisotropies in the focusing system, dispersion and bending, collimation, etc. An example of a nonequilibrium beam distribution is the S-G distribution introduced above. It can be used, for example, to model the wavelike beam evolution described by Bernal et al. [19]. A space-charge-dominated electron beam emerging from an aperture develops a ring of charge near its edge that progresses towards the beam's center as the beam propagates in a solenoid or quadrupole system. Simulations with WARP starting with a S-G distribution accurately reproduce the density modulation in the experiment. In a similar fashion, Kishek et al. [20] reports spacecharge modes, similar to those observed before, in simulations of nonequilibrium charged particle beams with anisotropy. We are interested in using tomography as a diagnostic tool to study nonequilibrium dynamics in spacecharge-dominated beams. In order to do that, we simulate the $7 \mathrm{~mA}$ beam starting with a SG distribution and observe the beam evolution by mapping its phase space. The phase space is recovered by scanning quadrupoles $Q 1, Q 2$, and $Q 3$, as described in Sec. III, downstream of Q3. Since we are interested in investigating beam propagation over a long distance, we add a series of alternating-gradient quadrupole (FODO) cells downstream of $Q 1$ with appropriate strength so that the beam remains matched as it passes through them. Our results are shown in Fig. 10, which demonstrates that tomography is powerful enough to capture the details of the distribution including the "wings" that correspond to the propagating space charge waves.

The sharp edges of the initial spatial distribution result in a strong force imbalance at the beam edges. As a consequence, the beam-edge particles experience forces that are very different from those affecting the bulk particles. Edge particles tend to move outside the beam as the latter is
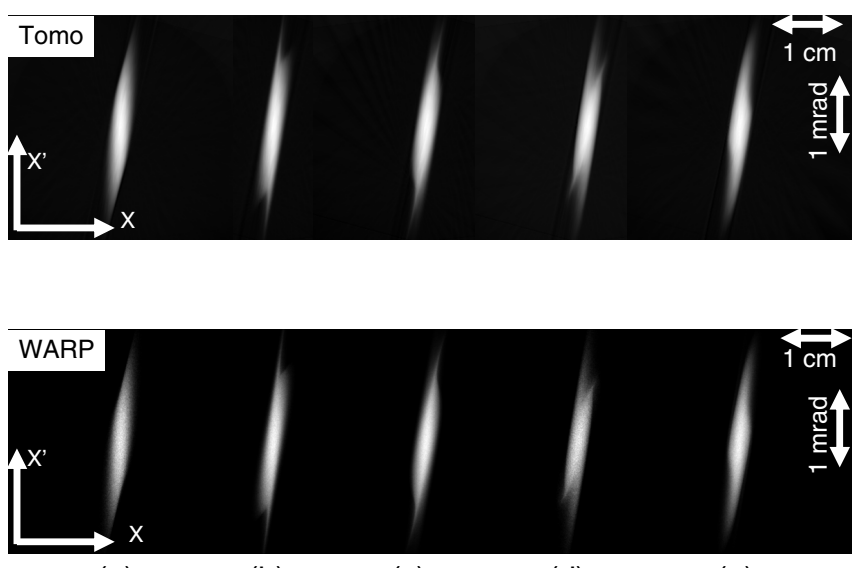

(a)

(b)

(c)

(d)

(e)

$\mathrm{Z}=45.2 \mathrm{~cm} \quad 77.2 \mathrm{~cm} \quad 109.2 \mathrm{~cm}$

$141.2 \mathrm{~cm} \quad 173.2 \mathrm{~cm}$

FIG. 10. Phase space from simulations of the $7 \mathrm{~mA}$ beam after propagating for different distances, clearly indicating the propagation of transverse density waves: tomography (top) vs direct WARP result (bottom) (cf. Ref. [19]). Phase space after passing through (a) 3; (b) 5; (c) 7; (d) 9; and (e) 11 quadrupoles.

TABLE II. Beam emittances at different positions along the beam line as calculated from the phase spaces generated by tomography and WARP.

\begin{tabular}{lrrrrr}
\hline \hline$Z(\mathrm{~cm})$ & 45.2 & 77.2 & 109.2 & 141.2 & 173.2 \\
$\varepsilon_{x}$, Tomo $(\mu \mathrm{m})$ & 13.7 & 15.2 & 14.8 & 14.1 & 15.7 \\
$\varepsilon_{x}$, WARP $(\mu \mathrm{m})$ & 14.5 & 14.7 & 14.5 & 15.0 & 14.8 \\
\hline \hline
\end{tabular}

transported in the FODO lattice [Figs. 10(c) and 10(d)]. However, the strong external focusing brings the edge particles back near the beam edge [Fig. 10(e)], creating a wavelike density modulation downstream. The quality of the tomography process can be established by its ability to capture the wavelike behavior on the recovered phase space, in good agreement with the WARP-generated phase spaces. Table II summarizes the emittance measurements as calculated by tomography and WARP. Beside the very good agreement between the WARP and tomographic phase spaces, the emittance values agree also very well with error close to $5 \%$.

\section{EXPERIMENTAL RESULTS}

In this section we present preliminary experimental results of phase space mapping in UMER using tomographic techniques. Our experiment will be along the injector line [21]. The phase space to be reconstructed is for the case when $Q 1$ is operated at $0.884 \mathrm{~A}$ and $Q 2$ and $Q 3$ are turned off. Details of the beam parameters are shown in Table III. We map the phase space by scanning $Q 1, Q 2$, and $Q 3$ according the discussion in Sec. III. A phosphor screen downstream of $Q 3$ displays the electron particle 
TABLE III. Experimental beam parameters.

\begin{tabular}{lc}
\hline \hline Beam energy & $10 \mathrm{keV}$ \\
$\beta(=v / c)$ & 0.2 \\
Beam current & $0.6 \mathrm{~mA}$ \\
Generalized perveance & $8.96 \times 10^{-6}$ \\
Aperture radius & $0.25 \mathrm{~mm}$ \\
Solenoid current & $5.56 \mathrm{~A}$ \\
Quadrupole peak gradient & $3.61 \mathrm{G} / \mathrm{cm}$ \\
\hline \hline
\end{tabular}

distribution (time integrated over many pulses) and the beam intensity pictures are captured with a charge coupled device (CCD) camera and then digitized and analyzed using associated hardware and software. While collecting the images, care was taken to avoid sensor saturation. The collimating of the electron gun aperture is set at $0.25 \mathrm{~mm}$ in order to produce a beam current of $0.6 \mathrm{~mA}$. To validate this, we measured the beam current with a fast Bergoz current transformer located between the second and third quadrupoles in the straight section. A set of six steering magnets were used to keep the beam aligned [22]. Steering dipoles and quadrupole currents were monitored and controlled using Lab View software.

The recovered phase space is shown in Fig. 11. Because of beam expansion outside the phosphor screen, our projections were limited within the $0-175^{\circ}$ range. As a verification of the reconstructed phase space we calculate the beam sizes from the phase space and compare it with the results from the spatial distribution. The comparisons are summarized in Table IV. Next, from the phase space distribution we calculate beam emittances by using Eq. (23). The results are $\varepsilon_{x}=5.8 \mu \mathrm{m}$ and $\varepsilon_{y}=6.8 \mu \mathrm{m}$ for $x$ and $y$,

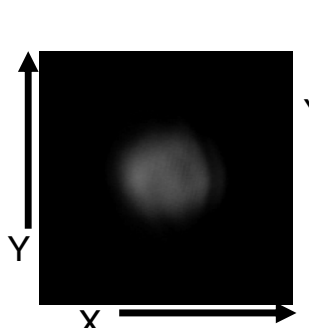

(a)

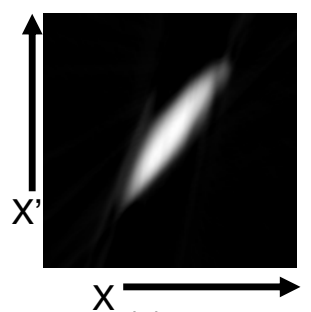

(c)

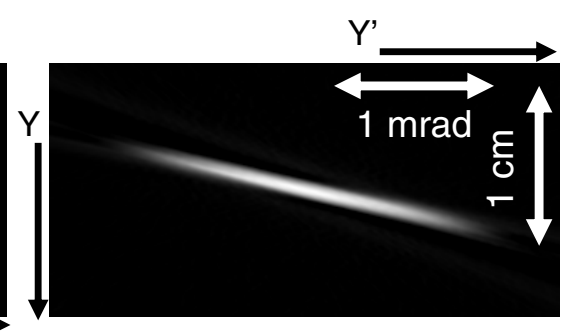

(b)
FIG. 11. Phase space tomography results along the UMER injector line: (a) spatial distribution at the point where recovery is done; (b) $Y^{\prime} Y$ tomographic recovery of the electron beam; (c) $X^{\prime} X$ tomographic recovery of the electron beam.
TABLE IV. Beam envelopes along $x$ and $y$ calculated from phase space and spatial distributions.

\begin{tabular}{lccc}
\hline \hline Distribution & Spatial & $x$-phase space & $y$-phase space \\
\hline$X(\mathrm{~mm})$ & 3.07 & 3.04 & \\
$Y(\mathrm{~mm})$ & 2.65 & & 2.49 \\
\hline \hline
\end{tabular}

respectively. It should be noted that the values of the emittance have errors that arise from the image processing analysis and uncertainty in the quadrupole currents. An independent way to measure emittance is by means of a quadrupole scan technique by curve-fitting rms beam sizes as a function of quadrupole current. A very good fit is obtained along the $x$-axis that gives $\varepsilon_{x}=6.0 \mu \mathrm{m}$ which is in good agreement with tomography. However, we were unable to estimate the emittance along the $y$-axis due to a very poor fit. Another way to estimate emittance for the pencil beam is by simply scaling it with aperture radius. The emittance and radius of the full beam behind the collimating aperture are $60 \pm 12 \mu \mathrm{m}$, and $3.2 \mathrm{~mm}$, respectively. Therefore, by assuming a uniform distribution at the aperture plate the emittance of the pencil beam is $4.7 \pm$ $1.0 \mu \mathrm{m}$. It should be noted that while scanning the quadrupoles the beam centroid was moving close to $1 \mathrm{~cm}$ in the vertical ( $y$ ) direction. Such large centroid errors could lead to nonlinear forces (for instance image forces) which increase errors, distort the beam, and can cause emittance growth. Such effects could explain the poor fit obtained from the quad scan and the higher emittance measured in the vertical direction; however, future experiments are needed in order to verify this.

\section{CONCLUSIONS}

Using the particle-in-cell code WARP we have simulated a UMER beam experiment and applied tomography techniques to map the phase space for beams in both emittanceand space charge-dominated regimes. For beams with space charge we are applying our tomography algorithm by assuming linear forces and no emittance growth. The validity of our reconstructed phase space was tested by comparing our results to the phase space generated directly by WARP (without tomography) which is highly accurate since it is not making the assumptions that our tomography algorithm does. For low current beams, we found excellent agreement between tomography and simulation. For more intense beams where space charge effects are present, still very good agreement exists, with the error close to $5 \%$. Furthermore, we applied tomography for beams with different, more complex, particle distributions. In each case, tomography reconstructs the beam phase space with equal accuracy; this indicates that the technique makes no assumption about the initial distribution. Finally, preliminary results of tomographic experiments performed on UMER were presented. 


\section{ACKNOWLEDGMENTS}

We wish to thank V. Yakimenko and I. Haber for useful discussions. We also wish to acknowledge helpful assistance regarding image analysis and camera hardware from R. Fiorito, R. Feldman, and D. Feldman. Finally we thank M. Holloway for reading the manuscript and making useful suggestions. The work is supported by the U.S. Department of Energy, the Office of Naval Research and the Joint Technology Office.

[1] J. Wei, Y. Y. Lee, D. Raparia, J. Sandberg, J. Tuozzolo, and W. T. Weng, "Spallation Neutron Source Ring-Status, Challenges, Issues, and Perspectives," Proceedings of the IEEE 2003 PAC Conference, Portland, OR, 2003, p. 571 .

[2] P. G. O'Shea and H. P. Freund, Science 292, 1853 (2001).

[3] M. Celata, Nucl. Instrum. Methods Phys. Res., Sect. A 544, 142 (2005).

[4] R. A. Kishek, G. Bai, S. Bernal, D. Feldman, T. F. Godlove, I. Haber, P. G. O'Shea, B. Quinn, C. Papadopoulos, M. Reiser, D. Stratakis, K. Tian, C. J. Tobin, and M. Walter, Nucl. Instrum. Methods Phys. Res., Sect. A 561, 266 (2006).

[5] P. G. O'Shea et al., Nucl. Instrum. Methods Phys. Res., Sect. A 464, 646 (2001).

[6] M. Reiser, Theory and Design of Charge Particle Beams (John Wiley \& Sons, Inc., New York, 1994).

[7] Claude Lejeune and Jean Aubert, Adv. Electron. Electron Phys. Suppl. 13A, 159 (1980).

[8] S. G. Anderson, J. B. Rosenzweig, G. P. LeSage, and J. K. Crane, Phys. Rev. ST Accel. Beams 5, 014201 (2002).

[9] L. A. Shepp and J. B. Kruskal, Am. Math. Mon. 85, 420 (1978).
[10] J. Radon, Math. Phys. Klasse 69, 262 (1917).

[11] C. B. McKee, P. G. O'Shea, and J. M. J. Madey, Nucl. Instrum. Methods Phys. Res., Sect. A 358, 264 (1995).

[12] V. Yakimenko, M. Babzien, I. Ben-Zvi, R. Malone, and X.-J. Wang, Phys. Rev. ST Accel. Beams 6, 122801 (2003).

[13] I. Ben-Zvi, J.X. Qiu, and X.J. Wang, "Picosecondresolution 'Slice' Emittance Measurement of ElectronBunches,' Proceedings PAC 1997, Vancouver.

[14] H. Li, Ph.D. dissertation, University of Maryland, College Park, 2004, pp. 102-117.

[15] A. Friedman, D. P. Grote, C. M. Celata, and J. W. Staples, Laser Part. Beams 21, 17 (2003).

[16] A. Friedman, Nucl. Instrum. Methods Phys. Res., Sect. A 544, 160 (2005).

[17] A. C. Kak, and M. Slaney, Principles of Computerized Tomographic Imaging (IEEE Press, New York, 1988).

[18] R. A. Kishek, S. Bernal, C. L. Bohn, D. Grote, I. Haber, H. Li, P. G. O'Shea, M. Reiser, and M. Walter, Phys. Plasmas 10, 2016 (2003).

[19] S. Bernal, R. A. Kishek, M. Reiser, and I. Haber, Phys. Rev. Lett. 82, 4002 (1999).

[20] R. A. Kishek, P. G. O'Shea, and M. Reiser, Phys. Rev. Lett. 85, 4514 (2000).

[21] T. Godlove, P. Haldemann, D. Kehne, S. Bernal, P. Chin, R. Kishek, Y. Li, M. Reiser, M. Venturini, J. G. Wang, W. W. Zhang, Y. Zou, and I. Haber, "The $10 \mathrm{keV}$ Injector for the University of Maryland Electron Ring Project," Proceedings of 1999 PAC, New York, NY, 1999.

[22] M. Walter, G. Bai, S. Bernal, I. Haber, M. Holloway, R. Kishek, P. O'Shea, B. Quinn, and M. Reiser, "Alignment and Steering for Injection and Multi-turn Operation of the University of Maryland Electron Ring (UMER)," Proceedings of 2005 PAC, Knoxville, TN, 2005. 